[2] M. Honig and M. Tsatsanis, "Adaptive techniques for multiuser CDMA receivers: Enhanced signal processing with short spreading codes," IEEE Signal Process. Mag., vol. 17, no. 3, pp. 49-61, May 2000.

[3] X. Wang, "Blind multiuser detection," in Encyclopedia of Telecommunications, J. G. Proakis, Ed. New York: Wiley, 2002.

[4] J. Hagenauer, E. Offer, and L. Papke, "Iterative decoding of binary block and convolutional codes," IEEE Trans. Inf. Theory, vol. 42, pp. 429-445, Mar. 1996.

[5] U. Fincke and M. Pohst, "Improved methods for calculating vectors of short length in a lattice, including a complexity analysis," Math. Comput., vol. 44, pp. 463-471, Apr. 1985.

[6] B. M. Hochwald and S. ten Brink, "Achieving near-capacity on a multiple-antenna channel," IEEE Trans. Commun., vol. 51, no. 3, pp. 389-399, Mar. 2003.

[7] H. Vikalo and B. Hassibi, "Toward closing the capacity gap on multiple antenna channels," in Proc. Int. Conf. Acoust., Speech, Signal Process., 2002, pp. 2385-2388.

[8] J. Boutros, N. Gresset, L. Brunel, and M. Fossorier, "Soft-input softoutput lattice sphere decoder for linear channels," in Proc. IEEE Global Commun. Conf., 2003.

[9] G. Schwarz, "Estimating the dimension of a model," Ann. Statist., vol. 6, pp. 461-464, 1978.

[10] P. Stoica and Y. Selén, "Model order selection-A review of information criterion rules," IEEE Signal Process. Mag., vol. 21, no. 4, pp. 36-47, Jul. 2004.

\section{Image Transmission Over the Polya Channel via Channel-Optimized Quantization}

Firouz Behnamfar, Fady Alajaji, and Tamás Linder

\begin{abstract}
We introduce two progressive methods for image transmission over binary channels with additive bursty noise modeled by the finite-memory Polya (contagion) channel. The methods, which are based on channel-optimized scalar quantization (COSQ) of the wavelet transform coefficients, exploit channel memory to offer superior performance over a number of more complex systems designed for the fully interleaved channel.
\end{abstract}

Index Terms-Channel optimized scalar quantization, channels with memory, convolutional codes, joint source-channel coding, subband coding.

\section{INTRODUCTION}

The traditional approach to data compression and channel coding is to treat these two tasks independently. The performance of the resulting systems - often called tandem systems — may be far from optimal when resources (computational/memory complexity and delay)are restricted. As

Manuscript received June 24, 2002; revised February 2, 2004. Part of the material in this correspondence was presented at the 2002 IEEE Data Compression Conference (DCC'02), Snowbird, UT, March 2002, and the 21st Biennial Symposium on Communications, Kingston, ON, Canada, June 2002. This work was supported in part by the Natural Sciences and Engineering Research Council (NSERC) of Canada and the Premier's Research Excellence Award (PREA) of Ontario. The associate editor coordinating the review of this paper and approving it for publication was Prof. Sheila S. Hemami.

F. Behnamfar is with the Department of Electrical and Computer Engineering, Queen's University, Kingston, ON K7L 3N6, Canada (e-mail: firouz@mast.queensu.ca).

F. Alajaji and T. Linder are with the Department of Mathematics and Statistics and the Department of Electrical and Computer Engineering, Queen's University, Kingston, ON K7L 3N6, Canada (e-mail: fady@mast.queensu.ca; linder@mast.queensu.ca).

Digital Object Identifier 10.1109/TSP.2004.840713 a result, it is beneficial to perform source compression and channel error protection jointly. This issue was quantitatively studied, for example, recently in [15], where joint source-channel coding is shown to outperform tandem coding if the systems have delay or complexity below a certain threshold. Several methods have been proposed for joint sourcechannel coding of images, including unequal error protection(UEP) [18], [21], [23], [26], [27] and channel-optimized scalar quantization (COSQ) [4]. In [26], image compression is performed through subband coding (with quadrature mirror filter (QMF) banks) followed by variable-length coding, and channel protection is done using packetization, check sum bits, and rate-compatible punctured convolutional (RCPC) codes. The approach in [23] is similar to [26], but the source coder is replaced with the strong method known as set partitioning in hierarchical trees (SPIHT) [22]. In [21], each subband is divided into a number of blocks. For every block, the bits at each level of significance are encoded with a different RCPC code rate. Finally, in [4], COSQ is used to transmit the transform coefficients. As COSQ is a fixed-length code, it does not suffer from the usual error propagation effect due to the use of lossless variable-length source codes. COSQ also combines source and channel coding; hence, it reduces the overall system complexity as compared with UEP methods.

In this correspondence, we present two COSQ-based image coders for transmission of images over noisy channels with memory. Memory is an important property of many real-life channels and is usually combated using channel interleaving (since most coding techniques are designed for memoryless channels). However, interleaving causes delay and increases complexity. Furthermore, the resulting associated memoryless channel has a lower capacity than the original channel with memory (for the broad class of information stable channels [1]). One salient feature of our COSQ methods is that they take into account the channel intra-block memory [19]; hence, their performance improves as channel noise becomes more correlated. Moreover, they provide reasonable image quality at bit rates as low as $0.125 \mathrm{~b} / \mathrm{pixel}$ and channel bit error rates (BERs) as high as 0.1 . They also outperform a typical UEP scheme that uses scalar quantization and convolutional coding (with and without channel interleaving).

In our systems, the source image is decomposed with discrete wavelet transform (DWT) or QMF banks into a number of subbands. As we begin the transmission of the data from the lower frequency subbands (and hence the reconstructed image quality improves as more data is received), the systems are progressive. For bit allocation among the subbands, we employ a generalized version of the technique in [28], which has low computational complexity. Finally, in the presence of channel mismatch between the design and actual channel BERs, the employed systems inherit the resilience of COSQ to outperform one of the strongest UEP methods that is introduced in [23]. When there is no channel mismatch, the UEP system of [23] (which employs interleaving) has a better performance than our methods; however, it has larger complexity and delay.

The rest of this correspondence is organized as follows. In Section II, the structure of our image transmission systems is described. In particular, the image decomposition, subband modeling, channel modeling, quantization, and bit allocation steps are addressed in detail. Simulation results are presented in Section III. Finally, conclusions are stated in Section IV.

\section{Image Coding For the Binary Polya Channel With Memory}

\section{A. Structure}

Fig. 1 shows the block diagram of the employed image coding systems which use the generic structure of [28]. It is assumed that the mean 


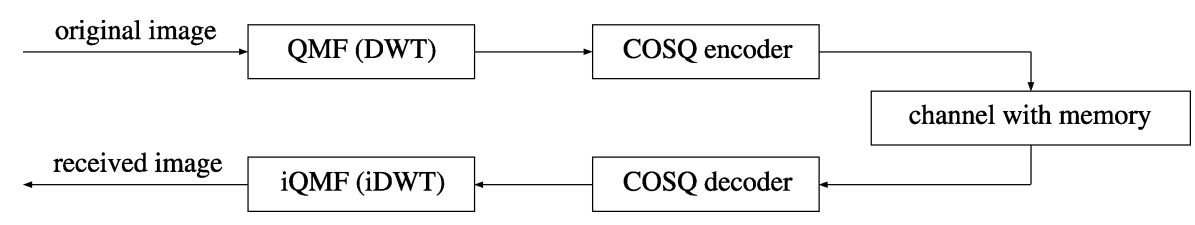

Fig. 1. Structure of the proposed image communication systems.

of the pixel intensities is removed. In the first method, the image is divided into a number of blocks. Every block is then transformed using the two-dimensional (2-D) DWT three times: every time on the lowest resolution level of the previous decomposition level. If the block size is $L \times L$ (we use $8 \times 8$ ), we next form $L^{2}$ subsources by grouping all $c_{i, j}^{(k)}, k=1, \ldots, K$ together, where $K$ is the number of blocks, and $c_{i, j}^{(k)}$ is the coefficient at row $i$ and column $j$ of block $k$. This method will be referred to as InterBlock. In the second method, the image is treated as one block. It is decomposed four times using the 32-D QMF banks of [14], resulting in 13 subbands, each of which is regarded as a subsource. We will refer to this system as IntraBlock. In both of these systems, the subsources are quantized using a COSQ for channels with memory with rates determined as explained in Section II-D. The resulting bit-stream is then sent directly over the channel. The receiver is simply the inverse of the transmitter.

For COSQ design, we need a good model for the distribution of the samples to be quantized. It is well known that the distribution of the coefficients of every subband is well approximated by the generalized Gaussian distribution [4], [26], with a probability density function given by

$$
f(x)=\frac{\alpha \eta(\alpha, \sigma)}{2 \Gamma\left(\frac{1}{\alpha}\right)} \exp \left\{-[\eta(\alpha, \sigma)|x|]^{\alpha}\right\}
$$

where $\eta(\alpha, \sigma)=1 / \sigma(\Gamma(3 / \alpha) / \Gamma(1 / \alpha))^{1 / 2}$ is the rate of decay, $\sigma^{2}$ is the variance, and $\Gamma(\cdot)$ is the Gamma function. For $\alpha=1$ and 2 , the above yields the Laplacian and Gaussian distributions, respectively. Based on our experiments on natural images and the results of [4] and [26], we assume here that the subsources in all subbands have the Laplacian distribution, and we quantize them using a COSQ trained for such a source. The benefit of subsource modeling is that the codebooks will not be image dependent and require no side information.

\section{B. Polya Contagion Model for Channels With Memory}

Most real-world communication channels suffer from correlated noise and/or fading distortions, often occurring in a bursty fashion. The most commonly used binary channel model to represent the equivalent discrete (hard-decision demodulated) fading channel with memory is the well-known Gilbert-Elliott channel (GEC), which was first introduced in [11] and later generalized in [7]. However, this channel model, which is based on a hidden Markov error process, is often difficult to mathematically analyze as it does not admit a closed-form expression for its channel capacity, and its block transition distribution is not transparently expressed in terms of the channel parameters. Recently, a simpler channel model assuming additive channel noise was introduced in [1], where the additive error process is generated by Polya's contagion urn scheme with finite memory. This noise process is a stationary ergodic Markov process of order $M$. To further motivate the selection of our channel model, we note that it has been recently shown (numerically) that the class of binary channels with additive $M$ th-order Markov noise (to which the channel in [1] belongs as a special case) is a good approximation (and, indeed, better than the GEC model) to hard-decision demodulated channels with correlated Rayleigh and Rician fading [20]. The Polya channel with $M=1$ is identical to an arbitrary channel with stationary ergodic first-order Markov noise; hence, it can approximate the Rayleigh fading channel in certain conditions [20]. We adopt the finite-memory Polya-contagion channel model in [1], which assumes that any noise sample depends only on the sum of the $M$ previous samples. If $X_{i}$, $Y_{i}$, and $Z_{i}$ represent the input, output, and noise in that order and $\oplus$ is addition modulo 2 , the channel input-output relationship is described by $Y_{i}=X_{i} \oplus Z_{i}$. Assuming that the input and noise are independent, for $i \geq M$ and any $e_{i-M}^{i-1} \in\{0,1\}^{M}$, we have [1]

$$
\operatorname{Pr}\left\{Z_{i}=1 \mid Z_{i-M}^{i-1}=e_{i-M}^{i-1}\right\}=\frac{\epsilon+\delta \sum_{j=i-M}^{i-1} e_{j}}{1+M \delta}
$$

where $\epsilon$ is the BER, and $\delta \geq 0$ controls the correlation coefficient of the noise given by $\delta /(\delta+1)$. The channel capacity (whose closed-form expression is derived in [1]) increases with $\delta$, suggesting that channel-optimized vector quantization (COVQ) may achieve less distortion for channels with memory. This intuition was empirically validated by [19], where a COVQ scheme was applied for the compression of ideal sources over this channel model. If $\delta$ is set to zero, the noise process becomes memoryless and the channel reduces to a binary symmetric channel (BSC). Note also that this model is less complex than the GEC model [17] and is completely specified with only three parameters $(\epsilon$, $\delta$, and $M)$. It has been recently adopted in several joint source-channel coding studies that include wireless applications (e.g., [5], [12], [13], and [25]).

\section{COSQ Design}

Let $d=d_{H}(\mathbf{x}, \mathbf{y})$ be the Hamming distance between the binary channel input block $\mathbf{x}=\left(x_{1}, \ldots, x_{n}\right)$ and the output block $\mathbf{y}=$ $\left(y_{1}, \ldots, y_{n}\right)$. The channel block transition probabilities are given by the following (see [1]):

- For $n \leq M, \mathrm{P}(\mathbf{y} \mid \mathbf{x})=L(n, d, \epsilon, \delta)$, where

$$
L(n, d, \epsilon, \delta)=\frac{\prod_{i=0}^{d-1}(\epsilon+i \delta) \prod_{i=0}^{n-d-1}(1-\epsilon+i \delta)}{\prod_{i=0}^{d-1}(1+i \delta)} .
$$

- For $n>M$

$$
\begin{aligned}
\mathrm{P}(\mathbf{y} \mid \mathbf{x})=L\left(M, s_{M+1}, \epsilon, \delta\right) & \\
& \times \prod_{i=M+1}^{n}\left[\frac{\epsilon+s_{i} \delta}{1+M \delta}\right]^{e_{i}}\left[1-\frac{\epsilon+s_{i} \delta}{1+M \delta}\right]^{1-e_{i}}
\end{aligned}
$$

where $e_{i}=x_{i} \oplus y_{i}$, and $s_{i}=e_{i-M}+\cdots+e_{i-1}$.

The significance of the above formulas is that unlike many other channel models in the literature, they provide easy and computationally inexpensive tools to implement the modified generalized Lloyd algorithm (GLA) for noisy channels [9].

Various algorithms have been proposed for COVQ design, among which, we tried the modified GLA initialized by simulated annealing [8], noisy channel relaxation [10], stochastic relaxation [29], and deterministic annealing [16]. After some experiments with the above algorithms, we decided to use the modified GLA with simulated annealing 
because of its computational efficiency. Unlike other methods, it computes the codebooks for rates as high as 8 bits per sample in a reasonable amount of time. In addition, the training distortion of the quantizers trained with this method were the most consistent with many trials (see Section III for more practical issues).

\section{Bit Allocation}

In subband coding of images, the end-to-end distortion is more sensitive to errors in the low-resolution subbands. Therefore, when allocating bits to the subsources, the subbands in which they are located should be taken into account. For the sake of brevity, in what follows, we explain the bit allocation strategy only for the IntraBlock method. Bit allocation for the InterBlock method is essentially the same.

Usually, the distortion of subsource $i$ is weighted by $w_{i}$, which is the $L_{2}$ norm of the basis functions of the subband to which it belongs (see, for example, [2] and [4] for the values of $w_{i}$ when DWT and QMF are used, respectively). Using the mean-square error distortion measure, we can write the end-to-end distortion as

$$
D=\sum_{i=1}^{S} w_{i} d_{i}, \quad d_{i}=\frac{1}{N_{i}^{2}} \sum_{m=1}^{N_{i}} \sum_{n=1}^{N_{i}}\left(c_{m, n}^{(i)}-\hat{c}_{m, n}^{(i)}\right)^{2}
$$

where $S$ is the number of the subsources (there are $S=13$ subsources in our implementation of the IntraBlock method), $\hat{c}_{m, n}^{(i)}$ is the reconstructed value of $c_{m, n}^{(i)}$, and we have assumed that the $i$ th subband has a size of $N_{i} \times N_{i} .{ }^{1}$ We employ dynamic programming for bit allocation. In particular, we extend the work in [28] for the Markov noise channel and for the case where the overall distortion has different sensitivities to different subsources. The bit allocation problem is to minimize $D$ in (1) subject to $\sum_{i=1}^{13} N_{i}^{2} r_{i} \leq B$ and $0 \leq r_{i} \leq r_{\max }$, where $r_{i}$ is the number of bits per sample allocated to the $i^{\text {th }}$ subsource, and $B$ is the total number of bits available. $r_{\max }$ is the maximum number of bits per sample that may be allocated to a subsource. Note that the overall bit rate is $(1 / R C) \sum_{i=1}^{13} N_{i}^{2} r_{i}$, where the image size is assumed to be $R \times C$. We choose $r_{\max }=8$ bits to have small codebooks and fast encoding. ${ }^{2}$ Modeling the subsources as independent Laplacian sources, we can write each $d_{i}$ in (1) as $\sigma_{i}^{2} d_{L}\left(r_{i}\right)$, where $d_{L}\left(r_{i}\right)$ is the distortion of a unit-variance Laplacian source quantized for a set of channel conditions (i.e., $\epsilon, \delta, M$ ), and $\sigma_{i}^{2}$ is the variance of the $i$ th subsource. The problem now is to allocate the available bits to 13 Laplacian sources, each with variance $w_{i} \sigma_{i}^{2}$, given the channel conditions. We use the algorithm in [28] to solve this problem, which is guaranteed to achieve the optimal bit allocation.

Note that $d_{L}\left(r_{i}\right)$ is calculated offline. In addition, although $\sigma_{i}^{2}$ is image-dependent, it is not computed inside the algorithm. For a 512 $\times 512$ image, the above algorithm requires a worst-case (unlikely) number of 262261 multiplications, 104 additions, and $3072 r$ comparisons for the calculation of the $\sigma_{i}^{2}$ and rate allocation, where $r$ is the overall bit rate. These figures change to 262720 multiplications, 512 additions, and $4032 r$ comparisons for the InterBlock method (see the Appendix for the number of operations in general). In both cases, the number of operations is very small compared with those required in UEP methods, where rate allocation forms a significant part of the computations (see, for example, [3] and [24]). ${ }^{3}$

\footnotetext{
${ }^{1}$ For the InterBlock method, the overall distortion can be written as $D=$ $\sum_{i, j=1}^{L} w_{i, j} d_{i, j}$, where $d_{i, j}=(1 / K) \sum_{k=1}^{K}\left(c_{i, j}^{(k)}-\hat{c}_{i, j}^{(k)}\right)^{2}$.

${ }^{2}$ Using a larger value for $r_{\max }$ will enhance the performance, but the training time and the size of the required codebooks will grow exponentially and tend to be prohibitive for $r_{\max }>8$ (given our computational resources).

${ }^{3}$ In order to reduce the complexity of rate allocation for such UEP methods, a parametric approach is developed in [18], where it is assumed that the logarithm of the block error rate has a linear relationship with the reciprocal of the channel code rate.
}

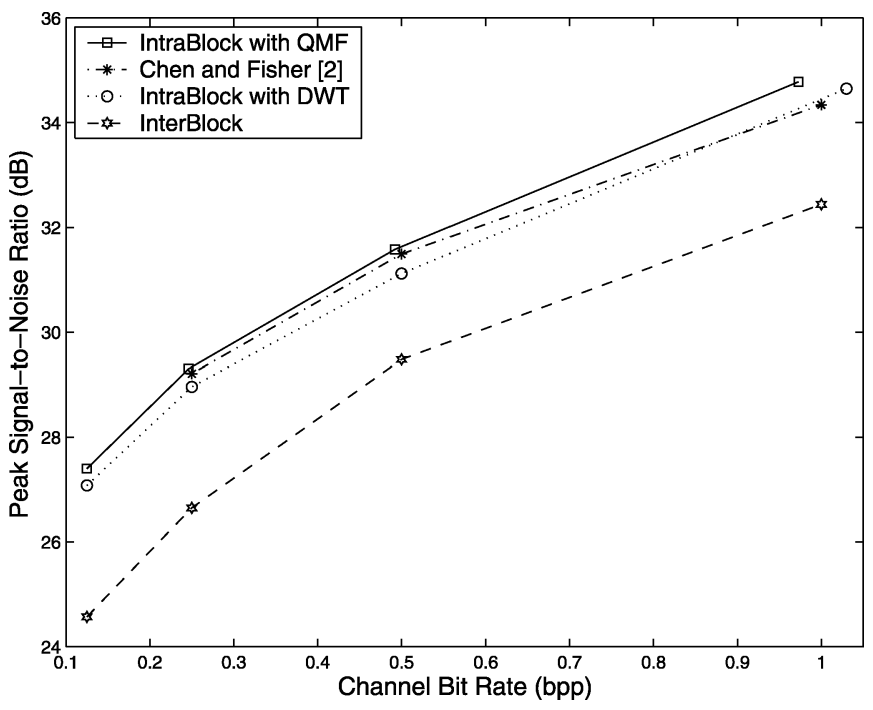

Fig. 2. Performance of various methods at $0.5 \mathrm{~b} /$ pixel, noiseless channel.

\section{E. Side Information}

Side information is the part of data in which no single bit error can be tolerated. Therefore, this amount must be kept as small as possible. ${ }^{4}$ The amount of side information of our IntraBlock method is identical to that in [4]. For a $512 \times 512$ image, the IntraBlock method needs the image mean, 13 sub-source variances, and the bit allocation table. Using 10, 10, and 3 bits for each of these amounts to a side information of $0.00068 \mathrm{~b}$ /pixel. For such an image and block size of $8 \times 8$, the InterBlock method has 64 subsources, and its side information becomes $0.0032 \mathrm{~b} / \mathrm{pixel}$. In comparison to the above figures, the method in [21] requires $0.07 \mathrm{~b} / \mathrm{pixel}$ of side information, assuming that 1.5 significance layers are encoded on average, and four different RCPC code rates are used.

\section{Simulation RESUlts}

We implemented the proposed image coder for the compression and transmission of gray-scale images over the contagion channel with $M=1$ and tested it for the image Lena (tests performed on other images such as Goldhill, Baboon, and Peppers gave results consistent with the Lena experiments). It takes around 20 hours to train the rate- 8 b/s COSQs with 200000 samples on Sun Ultra 60 machines. Over five trials, the training distortion was the same within $0.1 \mathrm{~dB}$.

Fig. 2 compares "IntraBlock with QMF," which is the IntraBlock method, "Chen and Fisher," which is the method in [4], "IntraBlock with DWT," whose structure is identical to "IntraBlock with QMF" but uses DWT, and InterBlock at an overall bit rate of $0.5 \mathrm{~b} /$ pixel for a noiseless channel. The performance of each system is measured in terms of the peak signal-to-noise ratio (PSNR), which is defined as PSNR $=10 \log _{10}\left(255^{2} / \sum_{i, j}\left(x_{i, j}-\hat{x}_{i, j}\right)^{2}\right)(\mathrm{dB})$, where $x_{i, j}$ and $\hat{x}_{i, j}$ are the original and reconstructed pixel values, respectively. For DWT, we used the 9/7 Daubechies filters, which are used in the JPEG2000 image compression standard [6]. Fig. 2 shows that IntraBlock (with QMF) outperforms the best reported COSQ-based method [4], which has exactly the same structure and filters as ours. This is due to our improved bit allocation and selection of the $w_{i}$ weights in (1). Note that the method in [4] is not suitable for channels with memory and requires channel interleaving. Fig. 2 also demonstrates that using

${ }^{4}$ We assume that the side information can be transmitted without errors using a very strong channel code. Since the amount of the side information is negligible as compared with the image information, this will cause a minimal increase in the overall bit rate. 


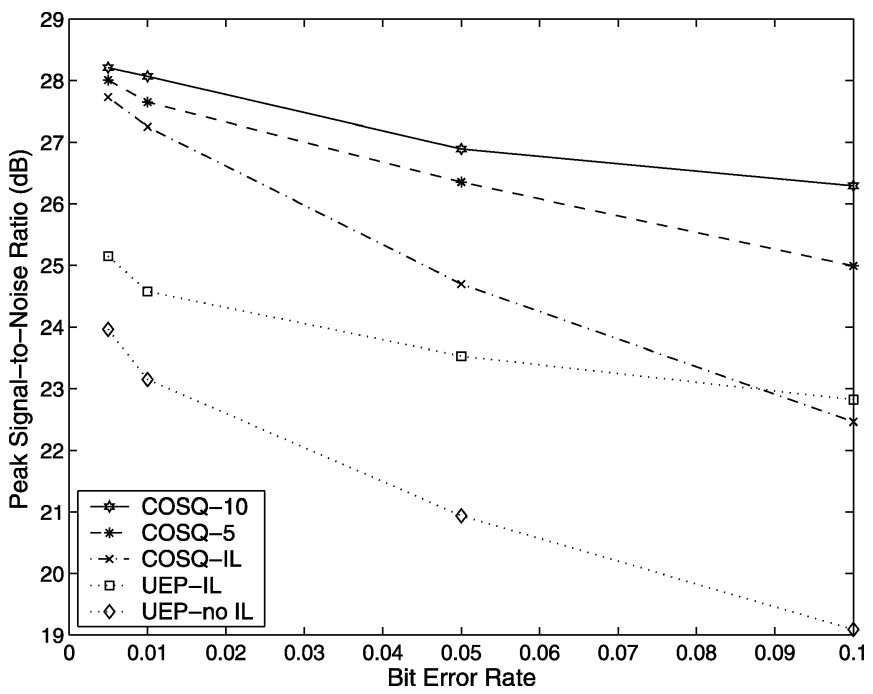

Fig. 3. Typical PSNR performance of various InterBlock methods over the binary channel with memory at $0.25 \mathrm{~b} /$ pixel. Block size is $8 \times 8$.

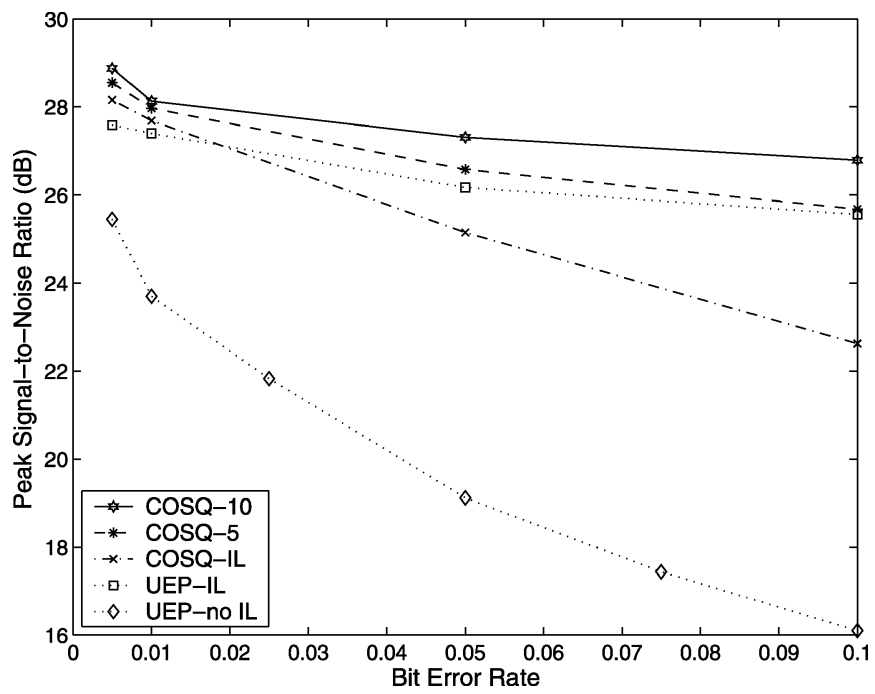

Fig. 4. Typical PSNR performance of various IntraBlock methods over the binary channel with memory at $0.25 \mathrm{~b} /$ pixel.

QMF banks yields better results than DWT filter banks (at the expense of higher arithmetic complexity).

Figs. 3 and 4 compare our methods in noisy channel conditions at a bit rate of $0.25 \mathrm{~b} /$ pixel with a typical UEP system, which is denoted by "UEP-IL" and comprises transform coding with QMF banks, scalar quantization of the transform coefficients, convolutional coding, and ideal channel interleaving (the plots for other bit rates show the same behavior). The convolutional codes have 64 states, are nonsymmetric, and are of rates $1 / 2\left(g_{11}=634, g_{12}=564, d_{\text {free }}=10\right)$, $1 / 3\left(g_{11}=574, g_{12}=664, g_{13}=744, d_{\text {free }}=14\right)$, and $1 / 4$ $\left(g_{11}=454, g_{12}=574, g_{13}=664, g_{14}=724\right.$, and $\left.d_{\text {free }}=20\right)$. The UEP system without the channel interleaver is also implemented and denoted by "UEP-no IL." The UEP systems choose the source and channel coding rates that maximize the PSNR for each set of the channel conditions. ${ }^{5}$ We refer to our system as COSQ, followed by the value of the channel correlation parameter $(\delta)$ for which it is designed (e.g., COSQ-5 and COSQ-10). COSQ-IL denotes the same system that uses an ideal channel interleaver $(\delta=0)$, and hence, it is designed for

${ }^{5}$ The source and channel coding rates were found to be $(1 / 8,1 / 2),(1 / 8,1 / 2)$, $(1 / 12,1 / 3)$, and $(1 / 16,1 / 4)$ for BER $=0.005,0.01,0.05$, and 0.1 , respectively.

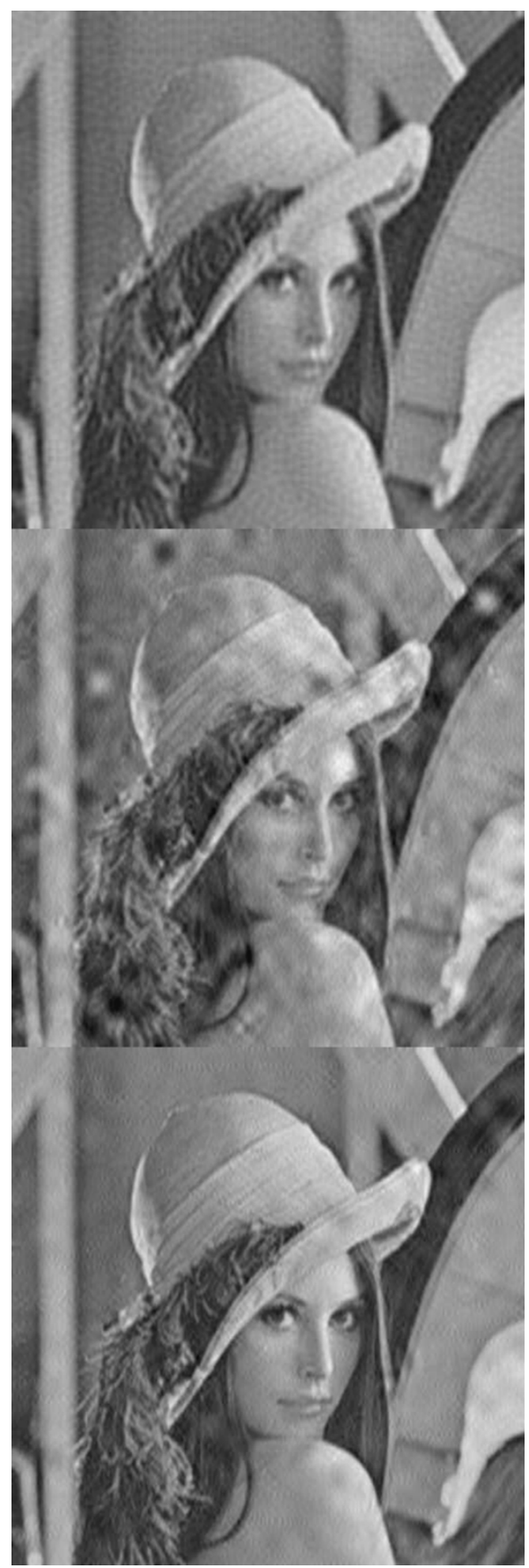

Fig. 5. Decoded image of various IntraBlock methods at $0.5 \mathrm{~b} /$ pixel and BER $=0.1$. From top to bottom: The best tandem coding scheme $($ PSNR $=27.4 \mathrm{~dB})$, COSQ-IL $($ PSNR $=23.5 \mathrm{~dB})$, and the COSQ-based system designed for noisy channels with memory (PSNR $=28.6 \mathrm{~dB}$ ).

the BSC with the same BER as the channel with memory. The plots show that the performance curves of the image coders designed for the correlated channel are higher than those of the interleaved channel. This demonstrates that substantial gains may be obtained from exploiting the channel memory instead of using interleaving, which increases delay and memory requirements. It is also observed that removing the channel interleaver to reduce the complexity of the UEP system (with the structure used here) would have catastrophic results. The plots also confirm the superiority of the IntraBlock over the InterBlock methods, as was seen in Fig. 2 for the noiseless channel.

The output image of three IntraBlock methods for the image Lena at a rate of $0.5 \mathrm{~b} /$ pixel is shown in Fig. 5. The figure shows that our 
TABLE I

BER Mismatch RESUlts IN TERMS OF PSNR IN DECIBELS FOR A RATE OF $0.5 \mathrm{~b} /$ pixel AND DESIGN BER OF 0.01

\begin{tabular}{l|c|c|c|c|c}
\hline Actual BER & 0 & 0.01 & 0.03 & 0.04 & 0.05 \\
\hline \hline IntraBlock & 31.15 & 30.43 & 29.18 & 28.79 & 28.25 \\
\hline InterBlock & 30.77 & 30.08 & 28.81 & 28.16 & 27.81 \\
\hline UEP method of [23] & 34.97 & 34.97 & 28.33 & 21.90 & 18.33 \\
\hline
\end{tabular}

method, which exploits channel memory, provides a higher quality decoded image than the systems that use channel interleaving.

Thus far, our image coding methods assume perfect knowledge of the channel parameters at both the transmitter and the receiver. However, in real-life situations, channel parameters are time-varying, resulting in channel mismatch. Table I compares our two coders in the presence of BER mismatch and that of Sherwood and Zeger [23], which is one of the best UEP schemes reported and is designed for the ideally interleaved channel (in [3] and [27], schemes that outperform [23] have been presented, but these works do not report on mismatch performance). It is observed that the IntraBlock system is the most robust against BER mismatch. Although the substantially more complex Sherwood-Zeger scheme performs better than ours by about $4 \mathrm{~dB}$ at no mismatch, it performs nearly $10 \mathrm{~dB}$ worse than our method in the presence of mismatch due to the variable-length lossless source code employed by that method. Resilience against mismatch is indeed a property of COSQ-based systems, which is inherited by our methods.

\section{CONCLUSION}

Two progressive image coding methods for the Polya contagion channel, which is a binary noisy channel with memory, are presented. Both systems are based on transform coding, subband modeling, and COSQ. Encoding an entire subband is observed to be better than dividing the image into blocks and encoding one coefficient from each block. The main result is that it is more beneficial to exploit the channel memory in the COSQ design, as opposed to suppressing its effect through channel interleaving, which also increases delay and complexity. COSQ was shown to outperform typical UEP schemes with or without channel interleaving. It is also observed that the performance of COSQ-based methods degrades gracefully (as is typical of such systems) in the presence of BER mismatch, whereas a powerful UEP method based on variable-length coding breaks down.

\section{APPENDIX}

In the following, we briefly explain the number of operations for rate allocation needed for our methods. In the IntraBlock method, $S=13$ subbands are formed. Then, the variance of the subbands are computed and multiplied by the training distortion of a Laplacian source, trained for the given channel conditions. Finally, the rate is allocated in a bit-by-bit fashion to the subband that maximizes the reduction in the overall distortion. In the InterBlock method, the image is first divided into $L \times L$ blocks ( $L=8$ in our case), and there are a total of $K$ blocks. Each subsource contains one coefficient from each block (at a given position). For both methods, the same rate allocation algorithm is used but with different input. The number of operations for each method is as follows.

IntraBlock Method

- $R C+S$ multiplications to find the variances $\sigma_{i}^{2}$, $i=1, \ldots, S$;

- $S \times r_{\max }$ multiplications to find $\sigma_{i}^{2} \times d_{L}\left(r_{i}\right), i=1, \ldots, S$, $r_{i}=1, \ldots, r_{\max }$;
- $S \times r_{\max }$ subtractions to find $\Delta D_{L}\left(r_{i}\right) \triangleq \sigma_{i}^{2}\left(d_{L}\left(r_{i}-1\right)-\right.$ $\left.d_{L}\left(r_{i}\right)\right), i=1, \ldots, S, r_{i}=1, \ldots, r_{\max } \cdot\left(d_{L}(0)=1\right)$;

- $S-1 \times R C r /((R / 16)(C / 16))=256(S-1) r$ comparisons to find the best sub-source to allocate one bit (one with maximum $\left.\Delta D_{L}\left(r_{i}\right), i=1, \ldots, S\right)$.

\section{InterBlock Method}

- $R C+L^{2}$ multiplications to find the variances $\sigma_{i}^{2}, i=$ $1, \ldots, L^{2}$

- $L^{2} \times r_{\max }$ multiplications to find $\sigma_{i}^{2} \times d_{L}\left(r_{i}\right)$, $i=1, \ldots, L^{2}, r_{i}=1, \ldots, r_{\max }$

- $L^{2} \times r_{\max }$ subtractions to find $\sigma_{i}^{2}\left(d_{L}\left(r_{i}-1\right)-d_{L}\left(r_{i}\right)\right)$, $i=1, \ldots, L^{2}, r_{i}=1, \ldots, r_{\max }$;

- $L^{2}-1 \times R C r /((R / L)(C / L))=L^{2}\left(L^{2}-1\right) r$ comparisons to find the best subsource to allocate one bit.

\section{REFERENCES}

[1] F. Alajaji and T. Fuja, "A communication channel modeled on contagion,” IEEE Trans. Inf. Theory, vol. 40, pp. 2035-2041, Nov. 1994.

[2] F. Behnamfar, F. Alajaji, and T. Linder, "Progressive image communication over binary channels with additive bursty noise," in Proc. Data Compression Conf., Snowbird, UT, Apr. 2002, pp. 272-281.

[3] V. Chande and N. Farvardin, "Progressive transmission of images over memoryless noisy channels," IEEE J. Sel. Areas Commun., vol. 18, pp. 850-860, Jun. 2000.

[4] Q. Chen and T. R. Fisher, "Image coding using robust quantization for noisy digital transmission," IEEE Trans. Image Process., vol. 7, pp. 496-505, Apr. 1998.

[5] Q. Chen and K. P. Subbalakshmi, "Joint source-channel decoding for MPEG-4 video transmission over wireless channels," IEEE J. Sel. Areas Commun., vol. 21, pp. 1780-1789, Dec. 2003.

[6] C. Christopoulos, A. Skodras, and T. Ebrahimi, "The JPEG2000 image coding system: an overview," IEEE Trans. Consumer Electron., vol. 46, pp. 1103-1127, Nov. 2000.

[7] E. O. Elliott, "Estimates of error rates for codes on burst-noise channels," Bell Syst. Tech. J., vol. 42, pp. 1977-1997, Sep. 1963.

[8] N. Farvardin, "A study of vector quantization for noisy channels," IEEE Trans. Inf. Theory, vol. 36, pp. 799-809, Jul. 1990.

[9] N. Farvardin and V. Vaishampayan, "On the performance and complexity of channel optimized vector quantizers," IEEE Trans. Inf. Theory., vol. 37, pp. 155-160, Jan. 1991.

[10] S. Gadkari and K. Rose, "Robust vector quantizer design by noisy channel relaxation," IEEE Trans. Commun., vol. 47, pp. 1113-1116, Aug. 1999

[11] E. N. Gilbert, "Capacity of burst noise channels," Bell Syst. Tech. J., vol. 39, pp. 1253-1265, Sep. 1960.

[12] R. Iordache, I. Tabus, and J. Astola, "Robust index assignment using Hadamard transform for vector quantization over finite-memory contagion channels," Circuits, Syst., Signal Process., vol. 21, pp. 483-507, Sep.-Oct. 2002.

[13] R. Iordache, I. Tabus, and A. Beghdadi, "Media-based post-processing for VQ image transmission over noisy channels," in Proc. Int. Symp. Signal Process. Applicat., Kuala Lumpur, Malaysia, Aug. 2001, pp. $48-51$.

[14] J. D. Johnston, "A filter family designed for use in quadrature mirror filter banks," in Proc. Int. Conf. Acoust., Speech, Signal Process., Denver, CO, 1980, pp. 291-294.

[15] J. Lim and D. L. Neuhoff, "Joint and tandem source-channel coding with complexity and delay constraints," IEEE Trans. Commun., vol. 51, pp. 757-766, May 2003.

[16] D. Miller and K. Rose, "Combined source-channel vector quantization using deterministic annealing," IEEE Trans. Inf. Theory, vol. 42, pp. 347-356, Feb./Mar./Apr. 1994

[17] M. Mushkin and J. Bar-David, "Capacity and coding for the GilbertElliott channel," IEEE Trans. Inf. Theory, vol. 35, pp. 1277-1290, Nov 1989.

[18] A. Nosratinia, J. Lu, and B. Aazhang, "Source-channel rate allocation for progressive transmission of images," IEEE Trans. Commun., vol. 51, pp. 186-196, Feb. 2003.

[19] N. Phamdo, F. Alajaji, and N. Farvardin, "Quantization of memoryless and Gauss-Markov sources over binary Markov channels," IEEE Trans. Commun., vol. 45, pp. 668-675, Jun. 1997. 
[20] C. Pimentel, T. H. Falk, and L. Lisbôa, "Finite-state Markov modeling of correlated Rician channels," in Proc. Canadian Workshop Inform. Theory, Waterloo, ON, Canada, May 2003, pp. 16-19.

[21] M. J. Ruf and J. W. Modestino, "Rate-distortion performance for joint source and channel coding of images," in Proc. Int. Conf. Image Process., vol. II, Washington, DC, Oct. 1995, pp. 77-80.

[22] A. Said and W. A. Pearlman, "A new, fast and efficient image codec based on set partitioning in hierarchical trees," IEEE Trans. Circuits Syst. Video Technol., vol. 6, pp. 243-250, Jun. 1996.

[23] P. G. Sherwood and K. Zeger, "Progressive image coding on noisy channels," in Proc. Data Compression Conf., Snowbird, UT, Mar. 1997, pp. 72-81.

[24] P. G. Sherwood, X. Tian, and K. Zeger, "Channel code block length and rate optimization for progressive image transmission," in Proc. Wireless Commun. Networking Conf., New Orleans, LA, Sep. 1999, pp. 978-982.

[25] K. P. Subbalakshmi and J. Vaisey, "Optimal decoding of entropy-coded Markov sources over channels with memory," in Proc. 33rd Annu. Conf. Inf. Sci. Syst., vol. 2, Mar. 1999, pp. 624-629.

[26] N. Tanabe and N. Farvardin, "Subband image coding using entropycoded quantization over noisy channels," IEEE J. Sel. Areas Commun., vol. 10, pp. 926-943, Jun. 1992.

[27] N. Thomos, N. V. Boulgouris, and M. G. Strintzis, "Wireless image transmission using turbo codes and optimal unequal error protection," in Proc. Int. Conf. Image Process., vol. I, Washington, DC, Sep. 2003, pp. 73-76.

[28] V. Vaishampayan and N. Farvardin, "Optimal block cosine transform image coding for noisy channels," IEEE Trans. Commun., vol. 38, no. 3, pp. 327-336, Mar. 1990.

[29] K. Zeger, J. Vaisey, and A. Gersho, "Globally optimal vector quantizer design by stochastic relaxation,” IEEE Trans. Signal Process., vol. 40, no. 2, pp. 310-322, Feb. 1992.

\section{Quasi-ML Period Estimation From Incomplete Timing Data}

Nicholas D. Sidiropoulos, Ananthram Swami, and Brian M. Sadler

\begin{abstract}
Given a noisy sequence of (possibly shifted) integer multiples of a certain period, it is often of interest to accurately estimate the period. With known integer regressors, the problem is classical linear regression. In many applications, however, the regressors are unknown integers, and only loose bounds on the period are available. Examples include hop period and timing estimation, wherein hops may be missed at the output of the frequency discriminator or the emitter may hop out of band; Pulse Repetition Interval (PRI) analysis; and passive rotating-beam radio scanning. We study several pertinent period estimators. Our emphasis is on a Quasi-Maximum Likelihood approach developed herein and an earlier method based on the Fourier Transform of a Dirac delta train representation of the data. Surprisingly, both are capable of attaining the clairvoyant Cramér-Rao Bound at moderate signal-to-noise ratios (SNRs), even for short (e.g., 10) samples. We carefully address parameter identifiability issues and corroborate our findings with extensive simulations.
\end{abstract}

Manuscript received July 10, 2003; revised February 23, 2004. This work was prepared through collaborative participation in the Collaborative Technology Alliance for Communications and Networks sponsored by the U.S. Army Research Laboratory under Cooperative Agreement DAAD19-01-2-0011. The associate editor coordinating the review of this paper and approving it for publication was Dr. Chong-Yung Chi.

N. D. Sidiropoulos is with the Departent of Elelctrical and Computer Engineering, Technical University of Crete, 73100 Chania, Crete, Greece, and also with the Departent of Elelctrical and Computer Engineering, University of Minnesota, Minneapolis, MN 55455 USA (e-mail: nikos@ telecom.tuc.gr).

A. Swami and B. Sadler are with the Army Research Laboratory, Adelphi, MD 20783-1197 USA (e-mail: aswami@ arl.army.mil; bsadler@arl.army.mil).

Digital Object Identifier 10.1109/TSP.2004.840761
Index Terms-Fourier transform, frequency estimation, missing data, period estimation, pulse repetition interval analysis, synchronization, timing estimation.

\section{INTRODUCTION}

Consider the following observation model:

$$
\tau(n)=\phi+\kappa(n) T+w(n), \quad n=1, \ldots, N
$$

where $\phi$ is an unknown shift, $\kappa(n) \in \mathbb{Z}$ is a generally unknown sequence of ordered integers, $T$ is the unknown period, and $w(n)$ is additive white Gaussian (AWG) noise, with variance $\sigma_{w}^{2}$. The problem is to estimate $\phi$ and $T$ from $\{\tau(n)\}$. In practice, there are many situations wherein the only information that can be assumed about the regressors is that $\kappa(n) \in \mathbb{Z}$, and perhaps also loose upper and lower bounds on $T$, or qualitative information of the type "lengthy gaps are rather rare."

The model in (1) is reminiscent of two well-known problems. In the special case that $\kappa(n)=n, n=1, \ldots N$, the problem is classical line regression; if the integers $\{\kappa(n)\}_{n=1}^{N}$ are known, then a standard linear regression problem appears. If the regressors $\{\kappa(n)\}_{n=1}^{N}$ are unknown integers, then a nonstandard regression problem emerges.

On the other hand, the problem in (1) is closely related to harmonic retrieval. That is, raising the data in (1) to the exponent yields

$$
x(n):=e^{j \tau(n)}=e^{j w(n)} e^{j(\phi+\kappa(n) T)}, \quad n=1, \ldots, N
$$

which is a harmonic retrieval problem with missing samples in nonGaussian multiplicative noise. Note, however, that raising the data to the exponent is not a reversible operation; hence, the problems are generally not equivalent.

The classical (single-) harmonic retrieval problem has been thoroughly investigated in the literature, including optimal (periodogram) and suboptimal linear-complexity solutions. The latter achieve nearoptimal performance at moderate signal-to-noise ratio (SNR) or moderate samples and above. Interestingly, Tretter [13] has shown that a computationally attractive solution can be obtained by casting the frequency estimation problem as a line regression problem in the phase domain. At high SNR, phase noise can be approximated by AWG noise, and the problems become essentially equivalent [13]. Another related approach to the problem of frequency estimation involves working with zero-crossings or higher order zero crossings of the observation [6], [11].

The harmonic retrieval problem with missing samples has also been considered [8]. Early approaches were periodogram-based (the periodogram often works reasonably well with mild multiplicative noise), but parametric techniques have also been developed [9]. In most cases, a simple Bernoulli miss model is adopted [9], [12]; otherwise it is assumed that missing samples occur periodically with known outage period. Harmonic retrieval in multiplicative noise has been dealt with (see, e.g., [4]), but to the best of our knowledge, harmonic retrieval in multiplicative noise and a deterministic unknown model for the missing samples has not been addressed in the literature.

The baseline for the present research is mostly the work of Fogel and Gavish [3], Sadler and Casey [1], [10], and Clarkson et al. [2], who also considered period estimation from the model in (1) with missing observations.

Fogel and Gavish [3] considered Maximum Likelihood (ML) period estimation from incomplete data for a certain convenient choice of the noise probability density function (pdf) that explicitly depends on the sought period. The said pdf is compactly supported, and hence, non- 